\title{
Análisis de Algoritmos Empleados en los Procesos de Igualación del Color para Películas Poliméricas
}

\author{
Ana C. Keiman ${ }^{(1)}$ y Rubén G. Barrera ${ }^{(2)}$ \\ Universidad Nacional Autónoma de México, (1) Instituto de Astronomía, (2) Instituto de Física, \\ AP 20-364, 01000, México D.F.-México (e-mail: ckeiman@astroscu.unam.mx)
}

Recibido Nov. 25, 2009; Aceptado Dic. 14, 2009; Versión Final recibida Dic. 30, 2009

\begin{abstract}
Resumen
Se analizó la relevancia de considerar la interfase aire-pintura en el proceso de igualación del color de una muestra y la importancia de tener una mejor caracterización de las pinturas que conforman la base de datos que se utiliza para reproducir el color de dicha muestra. El procedimiento de igualación del color en pinturas se basa en la teoría de Kubelka-Munk, en la cual, se presupone que el medio incidente es el mismo que el polímero de la pintura. El análisis se realizó igualando el color de una muestra mezclando pinturas coloreadas con un solo pigmento a una pintura blanca, y mezclando pinturas coloreadas con un solo pigmento a una pintura blanca formulada con pigmentos TiO2, de la cual se conocen los valores de $\mathrm{K}$ y S. Los cálculos fueron realizados con ayuda del software Mathemática 5.0. Se encontró que las coordenadas de color son sensibles a la interfase aire-pintura y que posiblemente un conocimiento más detallado de la formulación de pinturas permita obtener mejores resultados en la igualación del color.
\end{abstract}

Palabras clave: igualación del color, películas poliméricas, Kubelka-Munk

\section{Analysis in Color Matching algorithms for Polymeric film}

\begin{abstract}
The relevance of considering the air-paint interface in the color matching process of a sample and the importance of having a better characterization of the paintings that make up the database that is used to reproduce the color of the sample is analyzed. The procedure for color matching paints, whose formulation is unknown, is based on the Kubelka-Munk theory, which assumes that the incident medium is the same as the polymer of the paint. The analysis was performed by matching the sample's color by mixing colored paints with a single pigment to a white paint, mixing paint and colored with a single pigment to a white paint made with pigments $\mathrm{TiO}$, for which the values of $\mathrm{K}$ and $\mathrm{S}$ are known. Calculations were performed using the software Mathematica 5.0. In was found that the color coordinates are sensitive to the air-paint interface and possibly a more detailed understanding of the formulation of paints to obtain better results in the color matching.
\end{abstract}

Keyword: color matching, polymeric film, Kubelka-Munk 


\section{INTRODUCCION}

El control o reproducción del color es un tema importante para la industria pinturera, textil, alfarera, de baldosas, dental, etc. (Artigas et-al, 2002) En el caso particular de las pinturas de colores para paredes, uno de los objetivos que debe cumplir el formulador consiste en optimizar los procedimientos de igualación del color para crear pinturas que coincidan con el color de una muestra de formulación desconocida, ya que en éste tipo de pinturas, utilizadas generalmente con fines decorativos, lo que se busca es la coincidencia entre un color deseado y la pintura solicitada. (Wicks et-al, 1999)

Actualmente los algoritmos que permiten reproducir un color cualquiera se basan en la teoría de Kubelka-Munk, que corresponde al modelo más simple de transferencia radiativa. (Edström, 2007; Kubelka y Munk, 1931) Dichos procesos realizan todo el cálculo suponiendo la inexistencia de la interfase aire-pintura y no consideran los detalles de la formulación de la base de datos utilizada para igualar el color. Diversos estudios sobre propiedades ópticas de pinturas demuestran que considerar la interfase aire-pintura produce variaciones en el cálculo de la reflectancia de la pintura en cuestión. También se ha encontrado que la reflectancia esta ligada a la naturaleza del pigmento utilizado, es decir varía según el tipo, tamaño y forma del pigmento. (Curiel et-al, 2002; Gunde y Orel, 2000; Perera, 2005; Liu et-al, 2005)

Ninguno de estos resultados ha sido considerado en el proceso de igualación del color, (Bondioli etal, 2006; Ishikawa-Nagai et-al, 2005; Karbasi et-al, 2008) motivo por el cual, en este trabajo se analiza la variación del color de una pintura creada a partir de la mezcla de pinturas pertenecientes a una base de datos, cuya formulación es parcialmente conocida, y una base de datos de quien solo se conoce la reflectancia, realizando el proceso considerando o no la interfase aire-pintura, con el fin de igualar el color de una pintura muestra cuya formulación es desconocida.

Al calcular las diferencias $\Delta \mathrm{E}$ en términos de las coordenadas de color en el espacio Lab (Wyszecki y Stiles, 1982), y considerando que en el campo de las pinturas de pared el limite aceptable de variación debe estar entre 0 y 1.5,(Bondioli et-al, 2006) se encontró que las coordenadas de color son sensibles a la consideración o no de la interfase aire-pintura y que posiblemente un conocimiento más detallado de su formulación permita tener mayor control y mejores resultados al intentar reproducir un color.

\section{ALGORITMOS PARA LA IGUALACIÓN DEL COLOR}

\section{Algoritmo tradicional para igualar colores}

Como se mencionó en la introducción el procedimiento que generalmente se sigue para igualar el color en pinturas se basa en la teoría de Kubelka-Munk. Esta teoría presupone que: 1) la capa de pintura es tal que su extensión es infinita, lo que permite despreciar el efecto de los bordes; 2) la pintura está formada por un material homogéneo que presenta inhomogeneidades con dimensiones menores que el espesor de la película, con lo cual se asegura la existencia de esparcimiento; 3) el medio incidente y la pintura son de la misma especie y por lo tanto no existe interfase entre ellos desapareciendo las reflexiones externas e internas que se producen en la frontera entre ambos medios; y 4) la pintura es iluminada por un flujo entrante I, que representa el promedio de los rayos que viajan hacia el interior del material y refleja un flujo saliente $\mathrm{J}$, éste último definido como el promedio de los rayos que viajan hacia el exterior. La propagación de estos flujos esta caracterizada por los coeficientes de absorción (K) y esparcimiento (S) de Kubelka-Munk mediante las ecuaciones diferenciales (1) y (2). La reflectancia debida solo a la presencia de las inhomogeneidades incrustadas en la película para espesores infinitos viene dada por la ecuación (3). (Kubelka y Munk, 1931)

$$
\begin{aligned}
& d l=-(K+S) I d z+S J d z \\
& d J=(K+S) J d z-S I d z
\end{aligned}
$$

$\left.\mathrm{R}_{\mathrm{KM}}\right|_{\mathrm{z} \rightarrow \infty}=\left.\frac{\mathrm{J}}{\mathrm{l}}\right|_{\mathrm{z} \rightarrow \infty}=\frac{1}{\mathrm{a}+\mathrm{b}}=1+\frac{\mathrm{K}}{\mathrm{S}}-\sqrt{\left(1+\frac{\mathrm{K}}{\mathrm{S}}\right)^{2}-1}$ 
La manera tradicional en que los software de igualación de color proceden para igualar el color de cualquier pintura consiste en definir primero el valor K/S de la muestra y de las pinturas de la base de datos. Dicho cociente es calculado sustituyendo las reflectancias de las pinturas en cuestión, medidas por un espectrofotómetro, en la relación que resulta de despejar K/S de la ecuación (3). (Karbasi, et-al 2008)

Una vez caracterizadas las pinturas se procede a determinar las concentraciones y combinaciones necesarias de las pinturas de la base de datos, tal que la mezcla reproduzca el valor K/S de la muestra según la ecuación (4). (Artigas, et al., 2002; Billmeyer, et al., 1973a)

$$
\left(\frac{\mathrm{K}}{\mathrm{S}}\right)_{\text {Muestra }}=\sum_{\mathrm{i}=1}^{\mathrm{n}} \mathrm{f}_{\mathrm{i}}\left(\frac{\mathrm{K}}{\mathrm{S}}\right)_{\mathrm{i}}
$$

\section{Modificación al Algoritmo tradicional para igualar colores}

Nótese que el cálculo de K/S realizado en el proceso tradicional de igualación del color se hace suponiendo que no existe interfase aire-pintura, ya que el cociente se obtiene a partir de la ecuación (3). Pero, esta suposición esta lejos de ser cierta, por lo que para analizar el problema, acercándonos mas al fenómeno real, se propone efectuar el proceso de igualación del color considerando la forma en que un espectrofotómetro de esfera integradora mide la reflectancia de una pintura.

La esfera integradora de estos instrumentos son superficies recubiertas de un material blanco de gran pureza $\left(\mathrm{Ba}_{2} \mathrm{SO}_{4}\right)$ que refleja y esparce la luz de la fuente muy eficientemente. La esfera contiene cuatro orificios. En uno se coloca la muestra a analizar, en otro, la fuente que la iluminará, en un tercero se ubica el detector que recolectará la luz reflejada y en el cuarto orifico se tiene una trampa de luz que coincide con la dirección del haz reflejado de manera especular. (Molenaar et-al, 1999; Liu et-al, 2005)

Para disimular la situación en que un observador humano evaluaría la apariencia de una pintura, inclinando la muestra con respecto a sus ojos hasta lograr deshacerse del reflejo especular, se usa el espectrofotómetro en la geometría conocida como D/8 ${ }^{\circ}$ con Especular Excluido (EE). En la cual se ilumina la muestra con luz difusa en todas direcciones, se deja la trampa abierta para no contar con el reflejo especular y se recolecta la luz a $8^{\circ}$ con respecto de la normal de la muestra. Matemáticamente esta configuración queda expresada por la ecuación (5): (Curiel et-al, 2002)

$R_{E E}=\frac{\left(1-R_{\text {int }}\right) R_{K M}}{1-R_{\text {int }} R_{K M}}$

Donde $R_{K M}$ es la reflectancia de Kubelka-Munk de la ecuación (3) y $R_{\text {int }}$ corresponde a la reflectancia interna que se origina cuando los haces dentro de la pintura chocan con la interfaz de ésta con el aire, y se calcula mediante la ecuación (6): (Molenaar et-al, 1999; Liu et-al, 2005)

$\mathrm{R}_{\mathrm{int}}=1-\frac{1-\mathrm{R}_{\mathrm{ext}}}{\mathrm{n}_{2}^{2}}$

Donde $\mathrm{R}_{\text {ext }}$ es la reflectancia externa (Ecuación (7)) debida a la fracción de la radiación incidente que "rebota" en la frontera propagándose en dirección contraria al haz original y $\mathrm{n}_{2}$ es el índice de refracción del vehículo de la pintura. (Molenaar et-al, 1999; Liu et-al, 2005)

$R_{\text {ext }}=\frac{1}{2}+\frac{\left(n_{2}-1\right)\left(3 n_{2}-1\right)}{6\left(n_{2}+1\right)^{2}}+\frac{n_{2}^{2}\left(n^{2}-1\right)^{2}}{\left(n_{2}^{2}+1\right)^{3}} \ln \frac{\left(n_{2}-1\right)}{\left(n_{2}+1\right)}-2 n_{2}^{3} \frac{\left(n_{2}^{2}+2 n_{2}-1\right)}{\left(n_{2}^{2}+1\right)\left(n_{2}^{4}-1\right)}+\frac{8 n_{2}^{4}\left(n_{2}^{4}+1\right)}{\left(n_{2}^{2}+1\right)\left(n_{2}^{4}-1\right)^{2}} \ln \left(n_{2}\right)$

\section{METODOLOGÍA}

El estudio de la susceptibilidad de las coordenadas de color de una pintura obtenida mediante el proceso de igualación, estudiado en este trabajo, se analiza: 1) Sin considerar la interfase airepintura; y 2) Considerando la interfase aire-pintura. Para lo cual se elige una serie de pinturas 
muestras (PM_B1) a las que igualaremos su color al mezclar las pinturas de una base de datos integrada por 7 pinturas no caracterizadas (PBD_B5) y una pintura caracterizada (PBD_TiO2).

Las pinturas PBD_B5 de la base de datos y las pinturas PM_B1 fueron formuladas por el Centro de Investigación en Polímeros del grupo COMEX (CIP-COMEX), quienes nos proporcionaron las reflectancias medidas con un espectrofotómetro MINOLTA 3700d, y las coordenadas de color en el espacio Lab calculadas con el software del mismo instrumento. Sobre los detalles de su formulación únicamente se nos comentó que las PBD_B5 corresponden a pinturas de colores puras, es decir se crearon introduciendo pigmentos de colores al vehículo. En cambio, las PM_B1 se formularon agregando los mismos pigmentos a la pintura blanca PBD_B5, cuya concentración volumétrica de pigmentos blancos es de $16 \%$. Por lo que los cocientes $\mathrm{K} / \mathrm{S}$ de cada pintura se calcularon sustituyendo las reflectancias en la ecuación (8) para el caso 1 y en la ecuación (9) para el caso 2. Ambas ecuaciones se obtienen al despejar K/S de las ecuaciones (3) y (5) respectivamente. Por otro lado la pintura PBD_TiO2 corresponde a una pintura blanca con concentración volumétrica de 16\% de pigmentos de $\mathrm{Ti} \overline{\mathrm{O}} 2$-Rutilo formulada mediante el mismo programa, desarrollado en Mathematica 5.0 , con el que se realiza la igualación de color. Los valores de $\mathrm{K}$ y S se obtuvieron a través de las ecuaciones (10) y (11) al suponer que los pigmentos son esféricos permitiendo así usar la teoría de Mie (Bohren y Huffman, 1983).

$$
\begin{aligned}
& \left(\frac{K}{S}\right)_{\infty}=\frac{\left(R_{\infty}-1\right)^{2}}{2 R_{\infty}} \\
& \frac{K}{S}=\frac{\left(1-R_{\text {int }}\right)^{2}\left(R_{E E}-1\right)^{2}}{2 R_{E E}\left(1-R_{E E}+R_{\text {int }} R_{E E}\right)} \\
& K=\frac{3 f}{4 \pi a^{2}} C_{a b s} \\
& S=\frac{3 f}{4 \pi a^{2}} C_{\text {sca }}
\end{aligned}
$$

Para igualar el color de las PM_B1 se mezclaron las PBD_B5 de colores con la pintura blanca PBD_B5 (Mezcla A) y con la pintura blanca PBD_TiO2 (Mezcla B), utilizando la ecuación (4). Las concentraciones de las pinturas de la base de datos se eligieron de tal manera que el valor de la coordenada de color L de la mezcla coincidiera con el valor calculado para las PM B1 por el espectrofotómetro MINOLTA 3700d. Una vez calculadas las K/S de las mezclas se obtuvieron sus reflectancias utilizando las ecuaciones (3) y (5) para los casos 1 y 2 respectivamente, junto con las respectivas coordenadas de color en el espacio Lab. Finalmente, para analizar que tanto varían los colores para cada caso y cada mezcla se calculan las diferencias mediante la ecuación (12). (Wyszecki y Stiles, 1982)

$$
\Delta \mathrm{E}=\sqrt{\Delta \mathrm{L}^{2}+\Delta \mathrm{a}^{2}+\Delta \mathrm{b}^{2}}
$$

\section{RESULTADOS Y DISCUSIÓN}

En la Fig. 1 se exponen las reflectancias de las PM_B1 medidas por el CIP-COMEX. En las Fig. 2 y Fig. 3 se comparan los valores de las reflectancias y en las Fig. 4 y Fig. 5 de los cociente K/S calculados para las pinturas de las mezclas A y B formuladas siguiendo la metodología explicada anteriormente para los casos 1 y 2.

Las diferencias de color entre las PM_B1 y las distintas mezclas se muestran en la Tabla 1, donde $\mathrm{MA} / \mathrm{MB}$ se refiere al tipo de mezcla y $\mathrm{C} 1 / \mathrm{C} 2$ representa el caso con el cual fue calculada. Estas diferencias se obtuvieron a partir de las coordenadas de color en el espacio Lab (ecuación (12)) calculadas a partir de las reflectancias de las mezclas. Cabe mencionar que las coordenadas de color se obtuvieron considerando que las pinturas fueron iluminadas con el iluminante D65, el cual corresponde al espectro estándar que asemeja a luz del día. (Wyszecki y Stiles, 1982) 

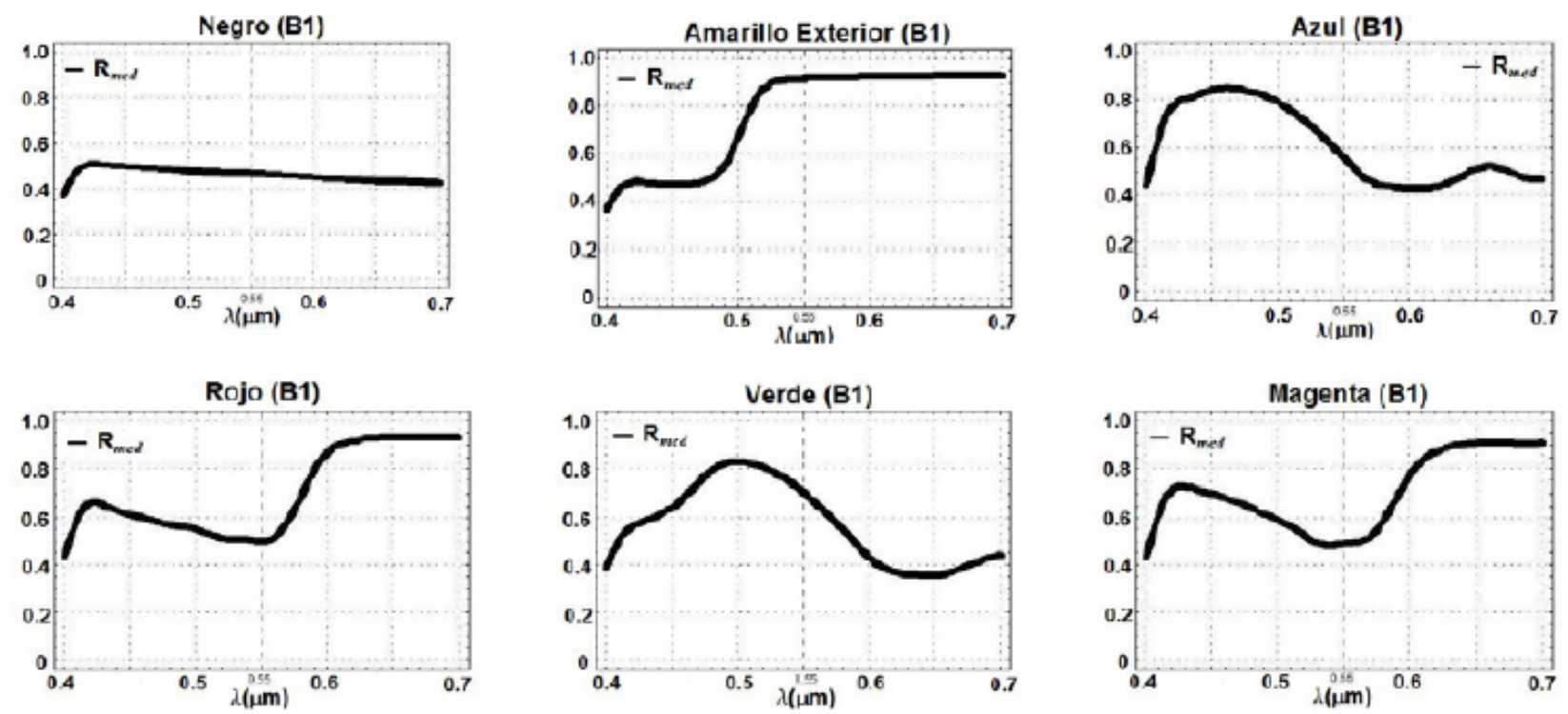

Fig. 1. Reflectancias de las pinturas muestras PM_B1 medidas por el CIP-COMEX
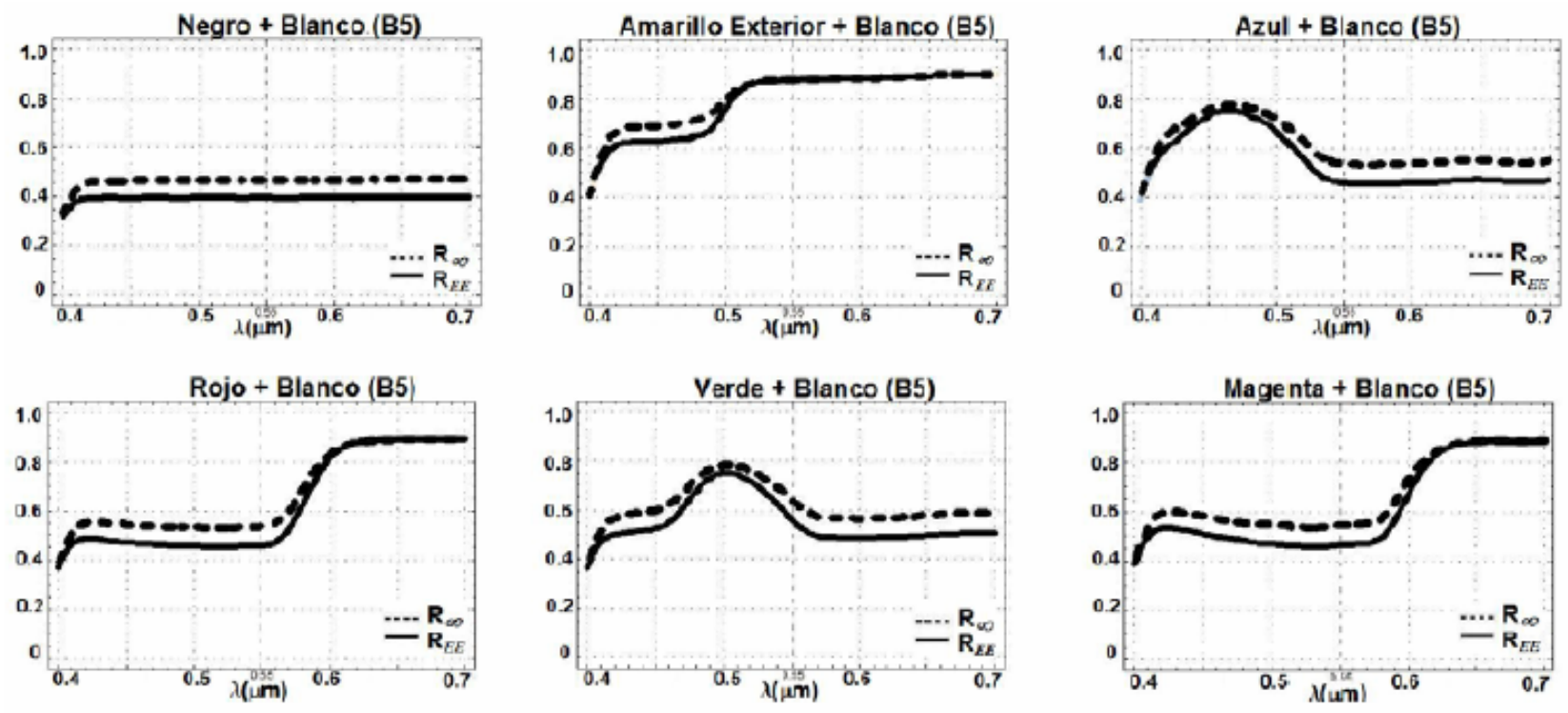

Fig. 2. Comparación de las reflectancias de las Mezclas A para los Caso 1 y 2
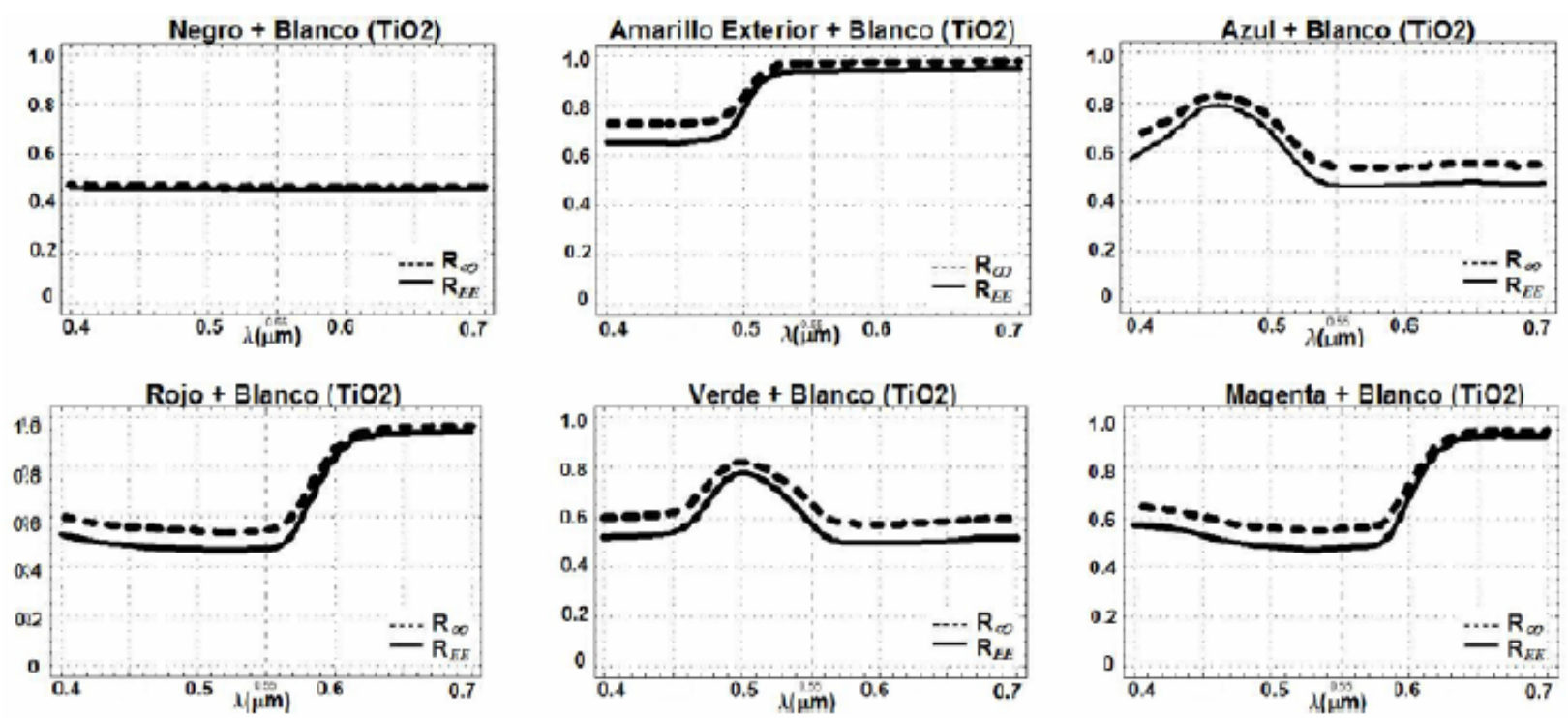

Fig. 3. Comparación de las reflectancias de las Mezclas B para los Caso 1 y 2 

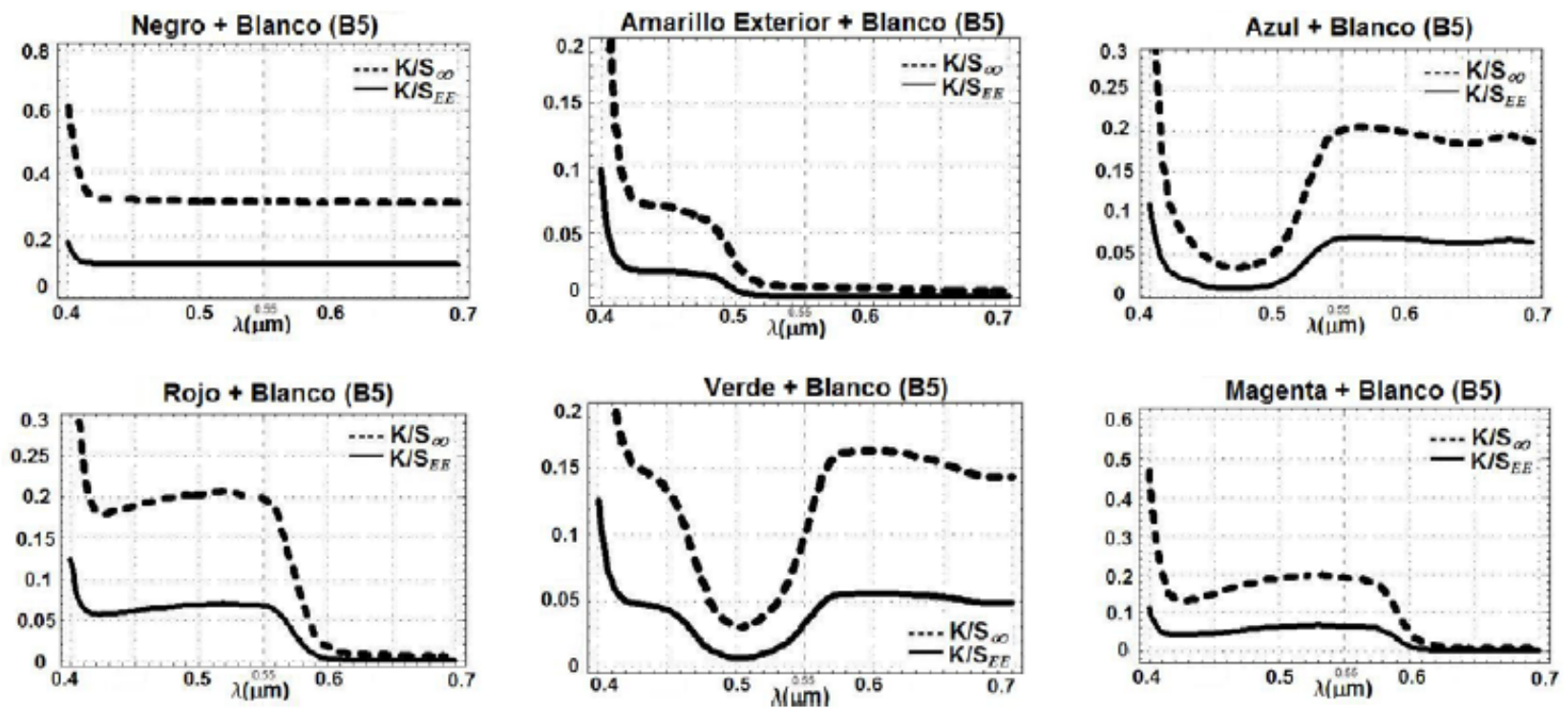

Fig. 4. Comparación de los valores K/S de las Mezclas A para los Caso 1 y 2
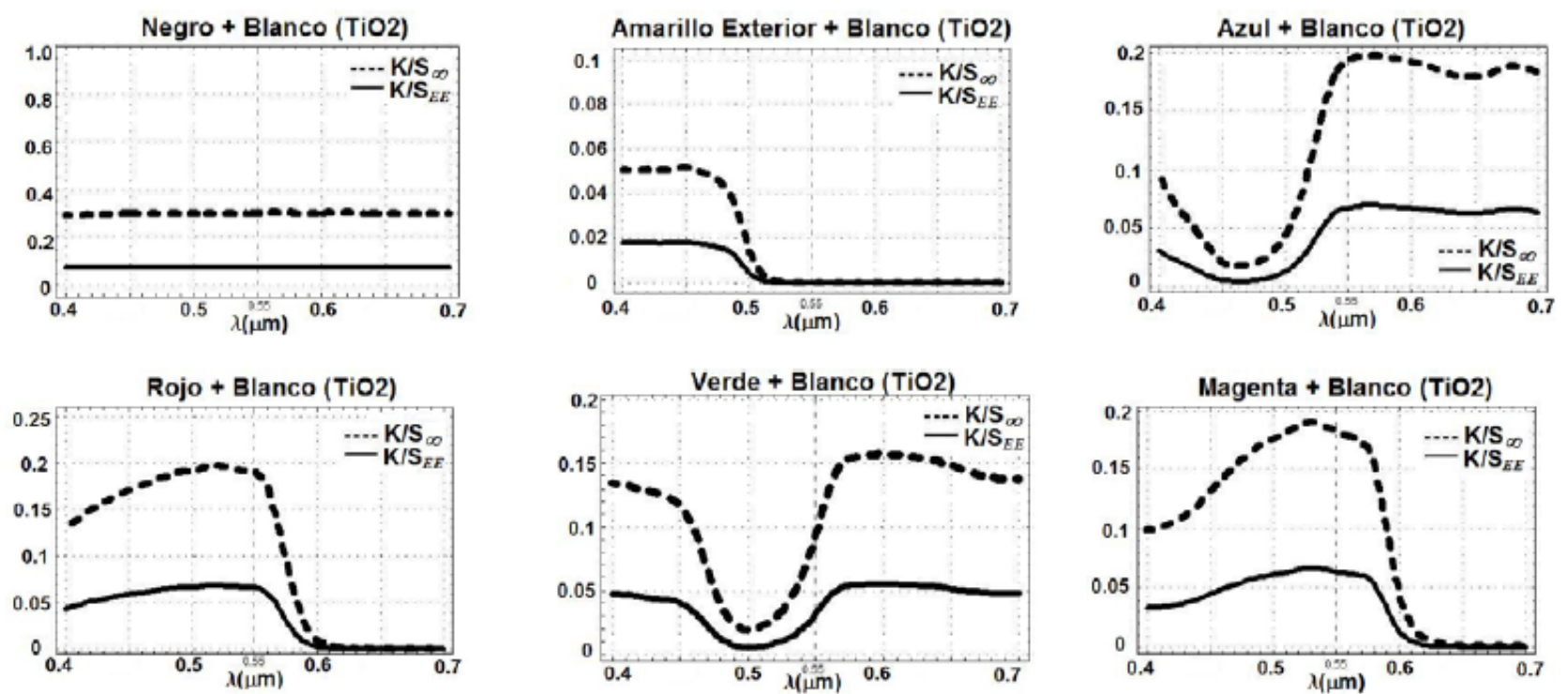

Fig. 5. Comparación de los valores $\mathrm{K} / \mathrm{S}$ de las Mezclas B para los Caso 1 y 2

Tabla 1. Diferencia de colores en el espacio Lab entre las pinturas PM_B1 y las mezclas A y B formuladas para los casos 1 y 2

\begin{tabular}{|c|c|c|c|c|}
\hline & \multicolumn{4}{|c|}{$\Delta \mathrm{E}$} \\
\hline & PM_B1 vs MA_C1 & PM_B1 vs MA_C2 & PM_B1 vs MB_C1 & PM_B1 vs MB_C2 \\
\hline Negro & 3.77 & 5.92 & 3.12 & 3.12 \\
\hline Amarillo & 19.59 & 15.31 & 17.78 & 13.82 \\
\hline Azul & 11.40 & 10.41 & 10.53 & 10.38 \\
\hline Rojo & 8.15 & 8.86 & 6.81 & 8.45 \\
\hline Verde & 13.89 & 11.15 & 12.87 & 10.49 \\
\hline Magenta & 11.04 & 10.68 & 9.42 & 9.83 \\
\hline
\end{tabular}

Observando las Figs. 2, 3, 4 y 5 se puede resaltar que las reflectancias y los valores K/S calculados, considerando la interfase aire-pintura, son menores a los obtenidos cuando se ignora dicha interfase. 
Por otro lado, si comparamos las reflectancias de las PM_B1 con las pinturas de las mezclas A (Fig. 1, Fig. 2) se distingue una similitud en la forma de las curvas, comportamiento que se pierde en el caso de las mezclas B. (Fig. 3) Con este resultado se podría confirmar que las PM_B1 fueron, en efecto, formuladas con el blanco de las PBD_B5. Pero nótese, que en ningún caso se reproducen exactamente las reflectancias deseadas. La consecuencia inmediata de esta falta de reproducibilidad en las reflectancias se refleja en el color de las pinturas creadas para igualar las PM_B1. Si bien, en la mayoría de los casos la diferencia pareciera imperceptible a simple vista, cuando se evalúa con un espectrofotómetro o cuando se utilizan otros iluminantes diferentes al D65, la variación del color puede llegar a ser muy notoria. Para hacer evidente este punto, basta con analizar los valores mostrados en la tabla 1 tomando en cuenta que la tolerancia de $\Delta \mathrm{E}$ es entre 0 y 1.5 y el error considerado en estos instrumentos es de $\Delta \mathrm{E} \sim 0.08$.

Para el caso de las pinturas amillara, azul y verde, la diferencia de color es menor cuando se toma en cuenta el efecto de la reflectancia interna (caso 2) independientemente de la mezcla usada. Pero, disminuye aún más si la igualación del color se realiza a partir de pinturas de las cuales conocemos los valores $\mathrm{K}$ y S, como es el caso de la mezcla B. Sin embargo, este comportamiento no lo vemos en las pinturas negra, roja y magenta, las cuales presentan una conducta errática que no permite definir un patrón de comportamiento.

Este comportamiento errático podría derivarse de haber utilizado la teoría de Kubelka-Munk para realizar los cálculos sin tomar en cuenta sus limitaciones, aun en el caso en que se considera la interfase aire-pintura. Dicha teoría esta desarrollada para situaciones en que la absorción es nula o muy pequeña, lo cual no es el caso de varios de los pigmentos usados en las pinturas de colores, por lo que suponemos que esta puede ser la razón por la cual no esté funcionando el proceso de igualación expuesto en este trabajo.

\section{CONCLUSIONES}

La reproducción del color de una pintura cualquiera resulta un problema complejo de tratar, como se demuestra en el presente trabajo. De los resultados expuestos se concluye que las coordenadas de color de pinturas formuladas a partir del proceso de igualación del color son sensibles a la consideración o no de la interfase aire-pintura, aunque no se pudo determinar un patrón de comportamiento.

Por otro lado, se hace evidente que la falta de información acerca de la formulación de las pinturas usadas en la base de datos (naturaleza de pigmentos, índice de refracción, tamaño, características del vehículo, etc.) nos impide atacar el problema de una manera controlada, pero sí nos permite determinar que para tener un mayor control en los resultados, el primer paso de cualquier formulador de pinturas es conocer a fondo las pinturas con las cuales hará sus mezclas.

Si se logra tener una mejor caracterización de las pinturas de la base de datos, o sea si se conocen sus $\mathrm{K}$ y S, y el comportamiento de la variación de los colores en las pinturas formuladas para igualar el color de una muestra es igual al presentado en este trabajo se propone cambiar la teoría utilizada por la de $\mathrm{N}$-flujos, para evitar las limitaciones que presenta la teoría de Kubelka-Munk.

\section{NOMENGLATURAS}

$\begin{array}{ll}\text { CIP-COMEX } & \text { Centro de Investigaciones en Polímeros del grupo COMEX } \\ \mathrm{CIE} & \text { Commission Internationale de I'Eclairage } \\ \mathrm{R}_{\mathrm{EE}} & \text { Reflectancia con Especular Excluido } \\ \mathrm{R}_{\mathrm{int}} & \text { Reflectancia Interna } \\ \mathrm{R}_{\mathrm{ext}} & \text { Reflectancia Externa } \\ \mathrm{K} & \text { Coeficiente de Absorción de Kubelka } \\ \mathrm{S} & \text { Coeficiente de Esparcimiento de Kubelka } \\ \mathrm{C} & \text { Sección eficaz }\end{array}$




\section{AGRADECIMIENTOS}

Los autores agradecen al Centro de Investigaciones en Polímeros del grupo COMEX y a los Drs Fernando Curiel y Adriana Gómez por las explicaciones, ayudas e informaciones proporcionadas durante el desarrollo de este trabajo. Ana C. Keiman fue apoyada por una beca de maestría del CONACYT.

\section{REFERENCIAS}

Artigas, J. M., P. Capilla, J. Pujol, Tecnología del color, 1-422, Universitat de valència, Valencia, España (2002)

Billmeyer, F., Abrams, W., Richard L., Predicting reflectance and color of paint films by Kubelka-Munk analysis. I. Turbid-Medium Theory, Journal of Paint Technology, 45(579), 23-30 (1973)

Billmeyer, F., Abrams, W., Richard L., Predicting reflectance and color of paint films by Kubelka-Munk analysis. II. Performance Test, Journal of Paint Technology, 45(579), 23-30 (1973)

Billmeyer, F., Abrams, W., Richard L., Predicting reflectance and color of paint films by Kubelka-Munk analysis. III. Effect of concentration errors on color for mixtures of one chromatic pigment with white, Journal of Paint Technology, 46(592), 31-38 (1974)

Billmeyer, F., Abrams, W., Richard L., Predicting reflectance and color of paint films by Kubelka-Munk analysis. IV. Kubelka-Munk Scattering Coefficient, Journal of Paint Technology, 48(616), 30-36 (1974)

Bohren, C.F., Huffman, D.R., Absorption and Scattering of light by small particles, $1^{\mathrm{a}}$ Edición, Ed. John Wiley and Sons, New York, EEUU (1983)

Bondioli, F., Manfredini, T., Romagnoli, M., Color matching algorithms in ceramic tile production, Journal of the European Ceramic Society: 26, 311-316 (2006)

Curiel, F., Vargas, W., Barrera R., Visible spectral dependence of the scatering and absorption coefficientes of pigmented coatings from inversion of diffuse reflectance spectra, Applied Optics, 41(28), 548-556 (2002)

Edström, P., Examination of the revised Kubelka-Munk theory: considerations of modeling strategies, Journal Optical Society of America A, 24(2), 548-556 (2007)

Gunde, M. K., Orel, Z. C., Absorption and scattering of light by pigmento particles in solar-absorbing paints, Applied Optics, 39(4), 622-628, (2000)

Ishikawa-Nagai, S., Ishibashi, K., Tsuruta, O., Weber, H. P., Reproducibility of tooth color gradation using a coputer color matching technique applied to ceramic restorations, The Journal of Prosthetic Dentistry, 93(2), 129-136 (2005)

Karbasi, A, S. Moradian, S. Asiaban, Improving the performance of computer color matching procedures, J. Opt. Soc. Am A: 25(9), 2251-2262 (2008)

Kubelka, P., F. Munk, Ein Beitrag zur Optik der Farbanstrich, Zeitschrift fr technische Physik: 12, 593601 (1931)

Liu, L., Gong, R., Huang, D., Nie, Y., Liu, C., Calculation of emittance of a coating layer with the kubelka-Munk tehory and the Mie-scattering model, Journal Optical Society of America A, 22(11), 2424-2429 (2005)

Molenaar, R, Bosch, J. J. T., Zijp, J., Determination of Kubelka-Munk scattering and absorption coefficients by diffuse illumination, Applied Optics: 38 (10), 2068-2077 (1999)

Perera, D. Y., Effect of pigmentation on organic coating characteristics, Progress in Organic Coatings, 50, 247-262, (2004)

Wicks, Z., Jones, F., Pappas, S. P., Organic Coating. Science and Technology, 2ª edición, 1-630, John Wiley and Sons, EEUU (1999)

Wyszecki, G., Stiles, W.S., Color Science: concepts and Methods, quantitative Data and Formulae, $2^{\mathrm{a}}$ edición, 646, John Wiley and Sons, EEUU (1982) 